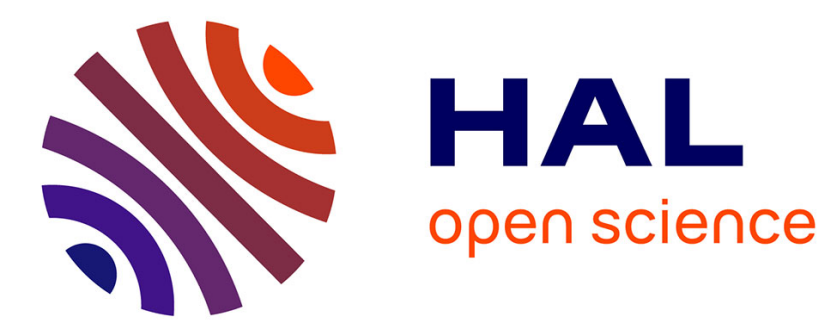

\title{
Equivalence between local tracking procedures and monotonic algorithms in quantum control
}

\author{
Gabriel Turinici
}

\section{To cite this version:}

Gabriel Turinici. Equivalence between local tracking procedures and monotonic algorithms in quantum control. CDC-ECC '05 - Proceedings of the 44th IEEE Conference on Decision and Control, and the European Control, Dec 2005, Seville, Spain. pp.8203-8208, 10.1109/CDC.2005.1583490 . hal00798230

\section{HAL Id: hal-00798230 \\ https://hal.science/hal-00798230}

Submitted on 11 Mar 2013

HAL is a multi-disciplinary open access archive for the deposit and dissemination of scientific research documents, whether they are published or not. The documents may come from teaching and research institutions in France or abroad, or from public or private research centers.
L'archive ouverte pluridisciplinaire HAL, est destinée au dépôt et à la diffusion de documents scientifiques de niveau recherche, publiés ou non, émanant des établissements d'enseignement et de recherche français ou étrangers, des laboratoires publics ou privés. 


\title{
Equivalence between local tracking procedures and monotonic algorithms in quantum control
}

\author{
Gabriel Turinici gabriel.turinici@inria.fr
}

\begin{abstract}
The computer simulations of quantum control use several approaches including local tracking procedures that prescribe the controlling field through the requirement that a certain functional be decreasing and monotonic algorithms that solve the Euler-Lagrange equations for a predefined cost functional. While different in implementation, recent works [1] hinted that these two classes share some common characteristics. We propose in this contribution a rigorous ground for such conclusions and discuss the precise formulation that allows to construct this equivalence.
\end{abstract}

\section{INTRODUCTION}

Manipulation of quantum phenomena was already demonstrated both in closed-loop laboratory experiments [2], [3], [4], [5], [6] and in theoretical studies on the controllability of quantum systems [7], [8], [9], [10].

Accompanying these advances, the computer simulations have the advantage to overcome experimental restrictions and have access to the whole dynamics allowing further insight and also providing hints in devising future experiments. Many algorithms have been proposed to solve the ensuing optimization problem among which two distinct classes can be identified. The first one contains the local tracking methods [11], [12], [13], [14], [15], [1] that propose explicit formulae of the driving field in an open-loop dependence on the evolving state. The formulae are obtained from the requirement to decrease a certain functional defined at each time instant and related to the "distance" to the target or by demanding strict adherence to a predefined observable trajectory. The second class are the monotonic algorithms [16], [17], [18] that solve the Euler-Lagrange equations associated to the optimization of a cost functional defined at a final time $T$. The two classes can also be combined as in [1].

Although different in implementation, these algorithms are shown below to be related in the sense that monotonic schemes are tracking procedures for some specific performance indexes. In the context of the density matrix formulation, this index is defined as the value of the cost functional (at the final time $T$ ) evaluated for the "best candidate" field at time $t \leq T$. This candidate field is made from the current field up to time $t$ that is prolonged with a given, reference field from $t$ to $T$ obtained at the previous iteration. This forward cost functional is decreased at each time instant. For the case of the wavefunction, due to the nonlinear nature of the target formulation, an upper bound is used to define the forward cost functional.

G. Turinici is with Inria Rocquencourt, Domaine de Voluceau, Rocquencourt B.P. 105, 78153 Le Chesnay Cedex, France and CERMICS-ENPC, Champs sur Marne, 77455 Marne la Vallée Cedex, France
The outline of the paper is the following: for the case of the density matrix we present the definition of tracking procedures in section II-A and one example of monotonic algorithm in section II-B. Then, we explain the relation between the two classes in sections II-C and II-D. The corresponding analysis for the wavefunction formulation is given in section III. Concluding discussions and remarks are the object of section IV.

\section{DENSITY MATRIX FORMULATION}

Consider a quantum system with internal dynamics described by the Hamiltonian $H_{0}$. Its interaction with an external (e.g., laser) field is modeled by introducing the dipole moment operator $\mu$ and the field intensity $\epsilon(t)$. If the system is represented in the density matrix formulation with initial state $\rho_{0}$ its dynamics will obey the time dependent Schrödinger equation:

$$
\begin{aligned}
& i \frac{\partial}{\partial t} \rho(x, t)=\left[H_{0}-\epsilon(t) \mu, \rho(x, t)\right] \\
& \rho(x, t=0)=\rho_{0}(x) .
\end{aligned}
$$

Here we used the convention $\hbar=1$.

We introduce the Liouville space representation, by defining the scalar product $\langle\langle a, b\rangle\rangle=\operatorname{Tr}\left(a^{\dagger} b\right)$ and the associated norm $\|a\|_{\rangle\rangle}=\sqrt{\langle\langle a, a\rangle\rangle}$. Instead of the commutators above, we define the operators $\mathcal{H}$ and $\mathcal{M}$ that act on density matrices by $\mathcal{H} \rho=\left[H_{0}, \rho\right], \mathcal{M} \rho=[\mu, \rho]$. Equation (1) becomes

$$
\begin{aligned}
i \frac{\partial}{\partial t} \rho(x, t) & =(\mathcal{H}-\epsilon(t) \mathcal{M}) \rho(x, t) \\
\rho(x, t=0) & =\rho_{0}(x) .
\end{aligned}
$$

The control goal can be expressed through the introduction of an observable operator $A$ by the requirement that the quantity $\langle A(t)\rangle=\operatorname{Re}\langle\langle A, \rho\rangle\rangle$ be maximized $(R e$ denoting the real part of a complex number). This formulation can be further refined as in [15] where an index $y(t)=y\left(\left\langle A_{1}(t)\right\rangle, \ldots,\left\langle A_{K}(t)\right\rangle\right)$ aggregating several observables is considered or even further by defining $y(t)=$ $y\left(\int_{0}^{t} \epsilon^{2}(s) d s,\left\langle A_{1}(t)\right\rangle, \ldots,\left\langle A_{K}(t)\right\rangle\right)$ where we introduce explicitly the dependence on the laser fluence. For notational convenience we will denote $F(t)=\int_{0}^{t} \epsilon^{2}(s) d s$.

\section{A. Tracking algorithm}

Consider the simple situation $y(t)=y\left(\int_{0}^{t} \epsilon^{2}(s) d s,\langle A(t)\rangle\right)$ where only one observable is considered i.e., $K=1$ above. We obtain

$$
\frac{d y(t)}{d t}=D_{1} y \cdot \epsilon(t)+D_{2} y \cdot \operatorname{Re}\left\langle\left\langle A, \frac{(\mathcal{H}-\epsilon(t) \mathcal{M}) \rho(x, t)}{i}\right\rangle\right\rangle
$$


where $D_{j}$ is the partial derivative with respect to the $j$-th variable. This can be further expressed as

$$
\frac{d y(t)}{d t}=f(F(t), \rho)+\epsilon(t) g(F(t), \rho)
$$

It is seen that, except for the points where $g$ vanishes (which will be called singularities and will be treated separately) for any desired trajectory $\widetilde{y}$ with $\widetilde{y}(0)=y(0)$, the condition $y(t) \equiv \widetilde{y}(t)$ uniquely determines the field $\epsilon(t)$ by the formula

$$
\epsilon(t)=\frac{\frac{d \widetilde{y}(t)}{d t}-f(F(t), \rho)}{g(F(t), \rho)} .
$$

From $d F / d t=\epsilon^{2}(t)$ one obtains that (4) is in fact a ODE on $F$ of the form

$$
d F / d t=\mathcal{Y}(F, \rho)
$$

that is to be solved jointly with (2) in order to ensure adherence to the prescribed trajectory $\widetilde{y}$.

Same considerations apply if only weaker properties are required, typically the increase/decrease of $y(t)$ which can be enforced through the condition $d y / d t \geq 0(\leq 0)$.

The difficulty in this approach is to find a suitable reference tracking trajectory $\widetilde{y}$ that does not give rise to singular points of the system (3), (5) i.e., where $g(F, \rho)=0$. In general singular points cannot be avoided a priori and techniques were designed to treat such situations: see [14] for designs that locally alter the trajectory to circumvent the singular points and [19], [1] for a study on the stopping points and procedures to improve their optimality.

\section{B. Monotonic algorithms for optimal control}

In an approach different from tracking, monotonically convergent algorithms pioneered in [20], [21] and extended in [18] in the wavefunction representation, are used in the context of the density matrix operator as in [22], [23]. Such procedures are included in the framework of the optimal control that introduces a cost functional (defined at a final time $T$ ) to be optimized. One such example of functional is

$$
J(\epsilon)=\int_{0}^{T} \alpha \epsilon^{2}(t) d t-2 R e\langle\langle A, \rho(T)\rangle\rangle .
$$

where $\alpha$ is a positive (constant or time varying) weight. Then, the critical points of $J(\epsilon)$ are sought after under the constraint of satisfying (2). Because of the constraint, a Lagrange multiplier, denoted $\chi(x, t)$ is introduced in the cost functional that now reads

$$
\begin{aligned}
& J_{2}(\epsilon)=\int_{0}^{T} \alpha \epsilon^{2}(t) d t-2 \operatorname{Re}\langle\langle A, \rho(T)\rangle\rangle \\
& +2 \operatorname{Re}\left\{\int_{0}^{T}\left\langle\left\langle\chi, \frac{\partial \rho}{\partial t}-\frac{(\mathcal{H}-\epsilon(t) \mathcal{M}) \rho}{i}\right\rangle\right\rangle d t\right\}
\end{aligned}
$$

The critical point equations are thus obtained:

$$
\begin{aligned}
& i \frac{\partial}{\partial t} \rho(x, t)=(\mathcal{H}-\epsilon(t) \mathcal{M}) \rho(x, t) \\
& \rho(x, t=0)=\rho_{0}(x) \\
& 2 \alpha \epsilon(t)+2 \operatorname{Re}\left\langle\left\langle\chi, \frac{\mathcal{M} \rho}{i}\right\rangle(t)=0\right. \\
& i \frac{\partial}{\partial t} \chi(x, t)=(\mathcal{H}-\epsilon(t) \mathcal{M}) \chi(x, t) \\
& \chi(x, T)=A .
\end{aligned}
$$

Building on these relations, the monotonic algorithms prescribe a particular order to iterate in these coupled equations by constructing, at the iteration step $k \rightarrow k+1$, a field $\epsilon_{k+1}(t)$ with the important property

$$
J\left(\epsilon_{k+1}\right) \leq J\left(\epsilon_{k}\right),
$$

hence the name of monotonic algorithm. A simple example of such algorithm is (see [22], [23] for additional details):

$$
\begin{aligned}
& i \frac{\partial}{\partial t} \rho_{k+1}(x, t)=\left(\mathcal{H}-\epsilon_{k+1}(t) \mathcal{M}\right) \rho_{k+1}(x, t) \\
& \rho_{k+1}(x, t=0)=\rho_{0}(x) \\
& \epsilon_{k+1}(t)=-\frac{1}{\alpha} \operatorname{Re}\left\langle\left\langle\chi_{k}, \frac{\mathcal{M} \rho_{k+1}}{i}\right\rangle(t)\right. \\
& i \frac{\partial}{\partial t} \chi_{k+1}(x, t)=\left(\mathcal{H}-\epsilon_{k+1}(t) \mathcal{M}\right) \chi_{k+1}(x, t)( \\
& \chi_{k+1}(x, T)=A
\end{aligned}
$$

This algorithm is proved [23] to have the convenient property in Eqn. (11). It is to be noted that this property is very surprising in this highly nonlinear setting, especially when considering that no second order information is directly involved in the computations.

Note that (12) and (13) are to be solved simultaneously because of the inter-dependence of the field $\epsilon_{k+1}(t)$ and the state $\rho_{k+1}(t)$. An alternative procedure is to insert relation (13) into equation (12) which will become a non-linear Schrödinger equation to be propagated forward in time.

Remark 1: Embedded into the writing of the scheme, at the convergence, the satisfaction of the critical point equations is ensured. See also [24] for further considerations on the convergence. Note that this desirable property is not always guaranteed for tracking.

\section{Forward cost functional}

Note that the cost functional of equation (6) has exactly the same minima and critical points as

$$
J_{\text {dist }}(\epsilon)=\int_{0}^{T} \alpha \epsilon^{2}(t) d t+\|A-\rho(T)\|_{\rangle\rangle^{2},}
$$

which measures the distance of the final density $\rho(x, T)$ to the target operator $A$. This conclusion is true due to the norm conservation properties of the Schrödinger equation which allows to write $J_{\text {dist }}=J+\|A\|_{\rangle\rangle}+\|\rho(T)\|_{\rangle\rangle}=J+\|A\|_{\rangle\rangle}+$ $\left\|\rho_{0}\right\|_{\rangle\rangle}$and thus to conclude that $J$ and $J_{\text {dist }}$ only differ by a constant.

The optimal control strategy of section II-B operates on a cost functional defined at final time $T$. As such, during the 
evolution at time $t<T$, this value is not yet accessible for immediate feedback into the optimization procedure. However, with a field computed up to $t<T$ a reasonable alternative is to use a candidate $\epsilon_{\text {ref }}$ on $[t, T]$ to compute the performance index at final time $T$. An appealing choice for $\epsilon_{r e f}$ is the field obtained at a previous iteration. We are thus lead to introduce for a control $\epsilon$ known on $[0, t]$ and a reference field $\epsilon_{r e f}$ defined on $[0, T]$ the field

$$
\bar{\epsilon}(s)= \begin{cases}\epsilon(s) & \text { for } 0 \leq s \leq t \\ \epsilon_{\text {ref }}(s) & \text { for } t \leq s \leq T .\end{cases}
$$

This field is the best available candidate at time $t<T$. Its performance index $J_{\text {dist }}(\bar{\epsilon})$ is

$$
J_{d i s t}(\bar{\epsilon})=\int_{0}^{t} \alpha \bar{\epsilon}^{2}(t) d t+\left\|A-\rho_{\bar{\epsilon}}(T)\right\|_{\rangle}{ }^{2},
$$

where $\rho_{\bar{\epsilon}}(T)$ is the state at time $T$ of the system

$$
\begin{aligned}
i \frac{\partial}{\partial t} \rho_{\bar{\epsilon}}(x, t) & =(\mathcal{H}-\bar{\epsilon}(t) \mathcal{M}) \rho_{\bar{\epsilon}}(x, t) \\
\rho_{\bar{\epsilon}}(x, t=0) & =\rho_{0}(x),
\end{aligned}
$$

A property with important practical implications on the efficient computation of $J_{\text {dist }}(\bar{\epsilon})$ is given in the following

Proposition 1: Define the forward cost functional for the control $\epsilon$ and reference field $\epsilon_{r e f}$ as

$$
\begin{aligned}
& J_{f w d}\left(\epsilon, t ; \epsilon_{\text {ref }}\right)=\int_{0}^{t} \alpha \epsilon^{2}(t) d t \\
& +\int_{t}^{T} \alpha \epsilon_{r e f}{ }^{2}(t) d t+\left\|\rho_{r e f}(t)-\rho(t)\right\|_{\rangle\rangle^{2}} .
\end{aligned}
$$

where $\rho$ evolves on $[0, t]$ as in (2) and $\rho_{\text {ref }}$ is the inverse propagation from $A$ with field $\epsilon_{r e f}$ :

$$
\begin{aligned}
& i \frac{\partial}{\partial t} \rho_{\text {ref }}(x, t)=\left(\mathcal{H}-\epsilon_{\text {ref }}(t) \mathcal{M}\right) \rho_{\text {ref }}(x, t) \\
& \rho_{\text {ref }}(x, t=T)=A .
\end{aligned}
$$

Then $J_{f w d}\left(\epsilon, t ; \epsilon_{\text {ref }}\right)=J_{\text {dist }}(\bar{\epsilon})$.

Proof The first two terms in $J_{f w d}\left(\epsilon, t ; \epsilon_{r e f}\right)$ are preciselly the first term of $J_{\text {dist }}(\bar{\epsilon})$. To compute the second term in $J_{d i s t}(\bar{\epsilon}), \rho$ is to be evolved from $\rho_{0}$ with the field $\bar{\epsilon}$ on $[0, T]$ to obtain $\rho(T)$. But, since $\rho_{\text {ref }}$ and $\rho$ evolve with the same field on the interval $[t, T]$, their distance will be constant throughout evolution and thus $\|A-\rho(T)\|_{\rangle}{ }^{2}=$ $\left\|\rho_{\text {ref }}(T)-\rho(T)\right\|_{\rangle}{ }^{2}=\left\|\rho_{\text {ref }}(t)-\rho(t)\right\|_{\rangle}{ }^{2}$. Thus we conclude that $J_{\text {dist }}(\bar{\epsilon})=J_{f w d}\left(\epsilon, t ; \epsilon_{\text {ref }}\right)$.

Remark 2: As the adjoint is available during the iterations of the monotonic algorithms, the above property can be used to monitor the evolution of the cost functional between two successive iterations. For instance this can help revealing which part of the evolution contributes more to the optimization and relate thus to local in time mechanisms of control.

\section{Monotonic algorithms as local tracking procedures}

The result above gives, at any intermediary time $t<T$ the value that the cost functional $J_{f w d}\left(\epsilon, t ; \epsilon_{\text {ref }}\right)$ will take at time $T$ if the optimization is stopped at the instant $t \leq T$ (and the field is put to be $\epsilon_{\text {ref }}$ on $[t, T]$ ). Note that the value of $J_{f w d}\left(\epsilon, t ; \epsilon_{r e f}\right)$ is readily computed at any time $t$ as soon as the inverse propagation (21) is computed once. Armed with this tool, optimization need not wait till the final time $T$ but can instead already operate at the current time $t$ using local tracking procedures to optimize the value $J_{f w d}\left(\epsilon, t ; \epsilon_{r e f}\right)$. We are now in position to claim the following

Theorem 1: The monotonic algorithm (12)-(14) is a local tracking procedure for the forward cost functional $J_{f w d}\left(\epsilon_{k+1}, t ; \epsilon_{r e f}=\epsilon_{k}\right)$ at any time $t$ in the sense that $J_{f w d}\left(\epsilon_{k+1}, t ; \epsilon_{k}\right)$ is a monotonically decreasing function of $t$ on the interval $[0, T]$.

Proof Note that $\epsilon_{k}=\epsilon_{\text {ref }}$ imply $\chi_{k}(t)=\rho_{\text {ref }}(t)$. Let us compute the time derivative of $J_{f w d}\left(\epsilon_{k+1}, t ; \epsilon_{k}\right)$ :

$$
\begin{aligned}
& \frac{d}{d t} J_{f w d}\left(\epsilon_{k+1}, t ; \epsilon_{k}\right)=\alpha \epsilon_{k+1}^{2}(t)-\alpha \epsilon_{k}^{2}(t) \\
& -2 \frac{d}{d t} \operatorname{Re}\left\langle\left\langle\chi_{k}(t), \rho_{k+1}(t)\right\rangle\right\rangle \\
& =\alpha \epsilon_{k+1}^{2}(t)-\alpha \epsilon_{k}^{2}(t) \\
& -2 \operatorname{Re}\left\langle\left\langle\frac{d}{d t} \chi_{k}(t), \rho_{k+1}(t)\right\rangle\right\rangle-2 \operatorname{Re}\left\langle\left\langle\chi_{k}(t), \frac{d}{d t} \rho_{k+1}(t)\right\rangle\right\rangle \\
& =\alpha \epsilon_{k+1}^{2}(t)-\alpha \epsilon_{k}^{2}(t) \\
& -2 \operatorname{Re}\left\langle\left\langle\frac{\left(\mathcal{H}-\epsilon_{k}(t) \mathcal{M}\right) \chi_{k}(t)}{i}, \rho_{k+1}(t)\right\rangle\right\rangle \\
& -2 \operatorname{Re}\left\langle\left\langle\chi_{k}(t), \frac{\left(\mathcal{H}-\epsilon_{k+1}(t) \mathcal{M}\right) \rho_{k+1}(t)}{i}\right\rangle\right\rangle \\
& =\alpha \epsilon_{k+1}^{2}(t)-\alpha \epsilon_{k}^{2}(t)+2 \operatorname{Re}\left\langle\left\langle\frac{\epsilon_{k}(t) \mathcal{M} \chi_{k}(t)}{i}, \rho_{k+1}(t)\right\rangle\right\rangle \\
& +2 \operatorname{Re}\left\langle\left\langle\chi_{k}(t), \frac{\epsilon_{k+1}(t) \mathcal{M} \rho_{k+1}(t)}{i}\right\rangle\right. \\
& =\alpha \epsilon_{k+1}^{2}(t)-\alpha \epsilon_{k}^{2}(t)+2 \epsilon_{k}(t) \alpha \epsilon_{k+1}(t) \\
& -2 \epsilon_{k+1}(t) \alpha \epsilon_{k+1}(t)=-\alpha\left[\epsilon_{k+1}(t)-\epsilon_{k}(t)\right]^{2} .
\end{aligned}
$$

Thus $J_{f w d}\left(\epsilon_{k+1}, t ; \epsilon_{k}\right)$ is a decreasing function of $t$.

Remark 3: The monotonicity follows as a corollary of the previous property of $J_{f w d}$, since $J_{d i s t}\left(\epsilon_{k}\right)=$ $J_{f w d}\left(\epsilon_{k+1}, 0 ; \epsilon_{k}\right) \geq J_{f w d}\left(\epsilon_{k+1}, T ; \epsilon_{k}\right)=J_{\text {dist }}\left(\epsilon_{k+1}\right)$.

The result above may also suggest the following interpretation: for any candidate solution $\epsilon_{k}$ two trajectories can be computed: $\rho_{k}(t)$ that starts from the correct initial condition $\rho_{0}$ but whose final state $\rho_{k}(T)$ may not yet be satisfactory close to the target, and the adjoint state $\chi_{k}(t)$ that propagates backward from the target $A$ but may not reach the correct initial state $\rho_{0}$; the idea is to make the trajectories coincide by computing $\epsilon_{k+1}$ such that $\rho_{k+1}(t)$ approaches monotonically $\chi_{k}(t)$. In the approximation where the fluence penalty $\int_{0}^{T} \alpha \epsilon^{2}(t)$ is negligible before the control part $\left\|\chi_{k}(t)-\rho_{k+1}(t)\right\|_{\rangle}$the distance between the two trajectories will decrease until its final value at time $T$. The situation is schematically depicted in Fig. 1. 


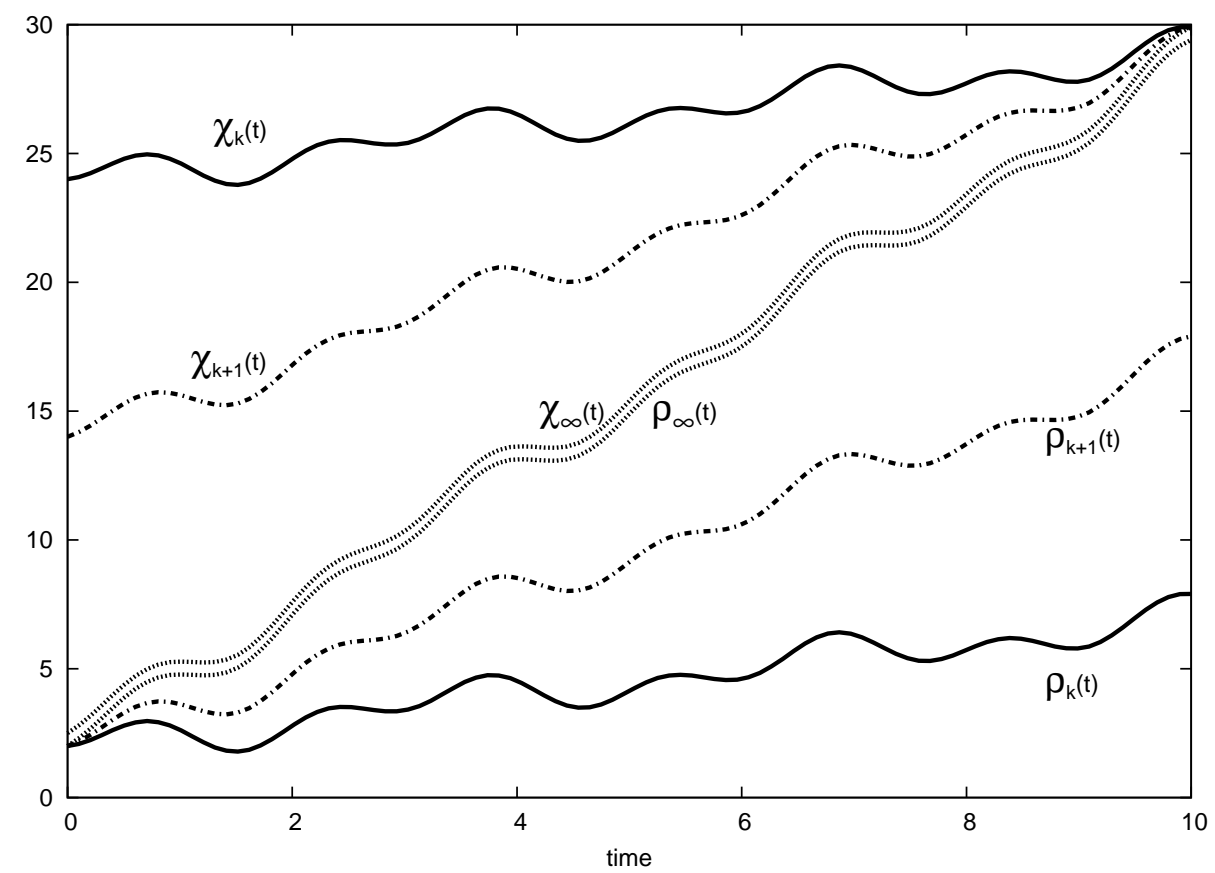

Fig. 1. Schematic illustration of the convergence of the monotonic algorithms for negligible fuence. The evolving state $\rho$ is +1 approaching monotonically the reference trajectory $\chi_{k}$. At the next iteration $\chi_{k+1}$ will remain at a constant distance from $\rho_{k+1}$ because both use the same fi eld $\epsilon_{k+1}$. This shrinking distance between the two trajectories ensure the progression of the cost functional toward optimal values. This observation is currently used in the context of effi cient parallelization of the numerical resolution of quantum control problems [25]. In the general case, the decreasing character of the distance between the curves is weighted by the fi eld fluence and the optimal couple of trajectories will be a tube whose nonzero width is related to the driving laser fi eld fluence.

\section{WAVEFUNCTION FORMULATION}

Similar considerations as introduced above apply to the wavefunction formulation. Note however that, even if the density matrix is more general than wavefunction, the associated observables are linear. For the wavefunction however, the observables enter into the cost functional as quadratic terms, which will induce some adaptations in the formalism. Let us consider the driving evolution equation:

$$
\begin{aligned}
& i \frac{\partial}{\partial t} \psi(t, x)=\left(H_{0}-\epsilon(t) \mu\right) \psi(t, x) \\
& \psi\left(t_{0}, x\right)=\psi_{0}(x) .
\end{aligned}
$$

For a given observable $A$, its averaged measured value is $\langle\psi|A| \psi\rangle$. With this definition the tracking formulation can be written as above and the same considerations apply.

\section{A. Monotonic algorithms}

Let us define the cost functional

$$
J^{w}(\epsilon)=\int_{0}^{T} \alpha \epsilon^{2}(t) d t-\langle\psi(T)|A| \psi(T)\rangle
$$

We introduce as before the adjoint state (Lagrange multiplier) $\chi(x, t)$ and give one example of monotonic algorithm [18]:

$$
\begin{aligned}
& i \frac{\partial}{\partial t} \psi_{k+1}(x, t)=\left(H_{0}-\epsilon_{k+1}(t) \mu\right) \psi_{k+1}(x, t) \\
& \psi_{k+1}(x, t=0)=\psi_{0}(x) \\
& \epsilon_{k+1}(t)=-\frac{1}{\alpha} \operatorname{Re}\left\langle\chi_{k}, \frac{\mu \psi_{k+1}}{i}\right\rangle(t) \\
& i \frac{\partial}{\partial t} \chi_{k+1}(x, t)=\left(H_{0}-\epsilon_{k+1}(t) \mu\right) \chi_{k+1}(x, t) \\
& \chi_{k+1}(x, T)=A \psi_{k+1}(T)
\end{aligned}
$$

We note that the nonlinearity in the observable induces a dependence of the adjoint state on the final state $\psi_{k+1}(T)$.

\section{B. Forward cost functional and equivalence}

We introduce, for a reference field $\epsilon_{\text {ref }}$ on $[0, T]$ and a field $\epsilon(s)$ defined up to an intermediary time $t<T$ the "candidate" solution $\bar{\epsilon}$ as in (16). It is possible to consider

$$
J^{w}(\bar{\epsilon})=\int_{0}^{T} \alpha \bar{\epsilon}^{2}(t) d t-\left\langle\psi_{\bar{\epsilon}}(T)|A| \psi_{\bar{\epsilon}}(T)\right\rangle
$$

with $\psi_{\bar{\epsilon}}$ evolving from $\psi_{0}$ with field $\bar{\epsilon}$. However, due to the nonlinear nature of the observable, no efficient procedure is available to compute $J^{w}(\bar{\epsilon})$ at time $t$ (other than explicitly computing $\psi_{\bar{\epsilon}}$ or working with the backward propagation of the observable $A$ with the field $\epsilon_{r e f}$ ). For this reason, monotonic algorithms use the following inequality for a positive definite observable $A \geq 0$

$$
-\langle a|A| a\rangle \leq-\langle b|A| b\rangle-2 \operatorname{Re}\langle a-b|A| b\rangle,
$$


(the difference of two quantities being $-\langle a-b|A| a-b\rangle \leq$ $0)$. In particular, if we denote by $\psi_{\epsilon_{r e f}}$ the state evolving from $\psi_{0}$ with field $\epsilon_{\text {ref }}$, one has

$$
\begin{aligned}
& J^{w}(\bar{\epsilon}) \leq \int_{0}^{T} \alpha \bar{\epsilon}^{2}(t) d t-\left\langle\psi_{\epsilon_{\text {ref }}}(T)|A| \psi_{\epsilon_{\text {ref }}}(T)\right\rangle \\
& +2 \operatorname{Re}\left\langle\psi_{\epsilon_{\text {ref }}}(T)-\psi_{\bar{\epsilon}}(T)|A| \psi_{\epsilon_{\text {ref }}}(T)\right\rangle
\end{aligned}
$$

It is therefore possible to define the (wavefunction ) forward cost functional

$$
\begin{aligned}
& J_{f w d}^{w}\left(\epsilon, t ; \epsilon_{\text {ref }}\right)=\int_{0}^{T} \alpha \bar{\epsilon}^{2}(t) d t-\left\langle\psi_{\epsilon_{\text {ref }}}(T)|A| \psi_{\epsilon_{\text {ref }}}(T)\right\rangle \\
& +2 \operatorname{Re}\left\langle\psi_{\epsilon_{\text {ref }}}(T)-\psi_{\bar{\epsilon}}(T)|A| \psi_{\epsilon_{\text {ref }}}(T)\right\rangle
\end{aligned}
$$

which can also be written

$$
\begin{aligned}
& J_{f w d}^{w}\left(\epsilon, t ; \epsilon_{\text {ref }}\right)=J^{w}\left(\epsilon_{\text {ref }}\right)+\int_{0}^{t} \alpha\left\{\epsilon^{2}(t)-\epsilon_{r e f}^{2}(t)\right\} d t \\
& +2 R e\left\langle\psi_{\epsilon_{\text {ref }}}(T)-\psi_{\bar{\epsilon}}(T)|A| \psi_{\epsilon_{\text {ref }}}(T)\right\rangle .
\end{aligned}
$$

To evaluate $J_{f w d}^{w}$ it is convenient to note that

$$
\begin{aligned}
& J_{f w d}^{w}\left(\epsilon, t ; \epsilon_{r e f}\right)=J^{w}\left(\epsilon_{r e f}\right)+\int_{0}^{t} \alpha\left\{\epsilon^{2}(t)-\epsilon_{r e f}{ }^{2}(t)\right\} d t \\
& +2 R e\left\langle\psi_{\bar{\epsilon}}(t)-\psi_{\epsilon_{\text {ref }}}(t), \chi_{\epsilon_{\text {ref }}}(t)\right\rangle .
\end{aligned}
$$

where $\chi_{\epsilon_{r e f}}(t)$ is the adjoint state at time $t$ given by

$$
\begin{aligned}
& i \frac{\partial}{\partial t} \chi_{\epsilon_{r e f}}(x, t)=\left(H_{0}-\epsilon_{r e f}(t) \mu\right) \chi_{\epsilon_{r e f}}(x, t) \\
& \chi_{\epsilon_{r e f}}(x, T)=A \psi_{\epsilon_{\text {ref }}}(T) .
\end{aligned}
$$

This formula allows an efficient computation of $J_{f w d}^{w}\left(\epsilon, t ; \epsilon_{\text {ref }}\right)$. To summarize, the cost functional $J^{w}(\bar{\epsilon})$ that cannot be computed explicitly is exploited through its upper bound $J_{f w d}^{w}\left(\epsilon, t ; \epsilon_{r e f}\right)$. The precise property of $J_{f w d}^{w}$ is given in the following

Theorem 2: The monotonic algorithm (25)-(27) is a local tracking procedure for the forward cost functional $J_{f w d}^{w}\left(\epsilon_{k+1}, t ; \epsilon_{\text {ref }}=\epsilon_{k}\right)$ at any time $t$ in the sense that $J_{f w d}^{w}\left(\epsilon_{k+1}, t ; \epsilon_{k}\right)$ is a monotonically decreasing function of $t$ on the interval $[0, T]$.
Proof. As above, we evaluate the time derivative of $J_{f w d}^{w}\left(\epsilon_{k+1}, t ; \epsilon_{k}\right)$.

$$
\begin{aligned}
& \frac{d}{d t} J_{f w d}^{w}\left(\epsilon_{k+1}, t ; \epsilon_{k}\right)=\alpha \epsilon_{k+1}^{2}(t)-\alpha \epsilon_{k}^{2}(t) \\
& +2 \frac{d}{d t} \operatorname{Re}\left\langle\psi_{k+1}(t), \chi_{k}(t)\right\rangle=\alpha \epsilon_{k+1}^{2}(t)-\alpha \epsilon_{k}^{2}(t) \\
& +2 \operatorname{Re}\left\langle\psi_{k+1}(t), \frac{d}{d t} \chi_{k}(t)\right\rangle+2 \operatorname{Re}\left\langle\frac{d}{d t} \psi_{k+1}(t), \chi_{k}(t)\right\rangle \\
& =\alpha \epsilon_{k+1}^{2}(t)-\alpha \epsilon_{k}^{2}(t) \\
& +2 \operatorname{Re}\left\langle\psi_{k+1}(t), \frac{\left(H_{0}-\epsilon_{k}(t) \mu\right) \chi_{k}(t)}{i}\right\rangle \\
& +2 \operatorname{Re}\left\langle\frac{\left(H_{0}-\epsilon_{k+1}(t) \mu\right) \psi_{k+1}(t)}{i}, \chi_{k}(t)\right\rangle \\
& =\alpha \epsilon_{k+1}^{2}(t)-\alpha \epsilon_{k}^{2}(t)+2 R e\left\langle\psi_{k+1}(t), \frac{\epsilon_{k}(t) \mu \chi_{k}(t)}{i}\right\rangle \\
& +2 R e\left\langle\frac{\epsilon_{k+1}(t) \mu \psi_{k+1}(t)}{i}, \chi_{k}(t)\right\rangle \\
& =\alpha \epsilon_{k+1}^{2}(t)-\alpha \epsilon_{k}^{2}(t)+2 \epsilon_{k}(t) \alpha \epsilon_{k+1}(t) \\
& +2 \epsilon_{k+1}(t) \alpha \epsilon_{k+1}(t)=-\alpha\left[\epsilon_{k+1}(t)-\epsilon_{k}(t)\right]^{2} .
\end{aligned}
$$

Thus $J_{f w d}^{w}\left(\epsilon_{k+1}, t ; \epsilon_{k}\right)$ is a decreasing function of $t$.

Remark 4: Note that the inequality $J^{w}(\bar{\epsilon}) \leq$ $J_{f w d}^{w}\left(\epsilon, t ; \epsilon_{r e f}\right)$ becomes equality in the limit where $\epsilon$ approaches $\epsilon_{\text {ref }}$ which will happen at the convergence of the monotonic algorithms as $\epsilon_{k+1}-\epsilon_{k} \rightarrow 0$.

Remark 5: As above, the monotonicity of the procedure is a simple consequence of the decrease of $J_{f w d}^{w}\left(\epsilon_{k+1}, t ; \epsilon_{k}\right)$ : $J^{w}\left(\epsilon_{k}\right)=J_{f w d}^{w}\left(\epsilon_{k+1}, 0 ; \epsilon_{k}\right) \geq J_{f w d}^{w}\left(\epsilon_{k+1}, T ; \epsilon_{k}\right) \geq$ $J^{w}\left(\epsilon_{k+1}\right)$.

\section{Conclusions}

Through the introduction of a "forward cost functional" this paper demonstrates that the monotonic algorithms are closely related to the class of tracking procedures. As such, the monotonicity property of the former algorithms appears as a natural consequence of the increasing/decreasing properties of the tracking index. The monotonic schemes are shown to construct at all intermediary times the cost functional value of a current " best field" candidate and use this information in the open loop to optimize the field further. For the specific case of the density matrix, this can also be interpreted using two trajectories that start/end at the correct states and whose distance is continuously reduced (depending also on the laser fluence) during the optimization process.

\section{ACKnOWLedgments}

The author gratefully acknowledge discussions on this topic with Mazyar Mirrahimi (ENSMP, Paris) and Julien Salomon (Paris VI University).

\section{REFERENCES}

[1] M. Mirrahimi, G. Turinici, and P. Rouchon, 'Reference trajectory tracking for locally designed coherent quantum controls," J. of Physical Chemistry A, 2005, to appear.

[2] R. Judson and H. Rabitz, "Teaching lasers to control molecules," Phys. Rev. Lett, 1992 
[3] A. Assion, T. Baumert, M. Bergt, T. Brixner, B. Kiefer, V. Seyfried, M. Strehle, and G. Gerber, "Control of chemical reactions by feedbackoptimized phase-shaped femtosecond laser pulses," Science, vol. 282, pp. 919-922, 1998.

[4] T. Weinacht, J. Ahn, and P. Bucksbaum, "Controlling the shape of a quantum wavefunction," Nature, vol. 397, pp. 233-235, 1999.

[5] R. Bartels, S. Backus, E. Zeek, L. Misoguti, G. Vdovin, I. P. Christov, M. M. Murnane, and H. C. Kapteyn, 'Shaped-pulse optimization of coherent emission of high-harmonic soft X-rays," Nature, vol. 406, pp. 164-166, 2000.

[6] R. J. Levis, G. Menkir, and H. Rabitz, "Selective bond dissociation and rearrangement with optimally tailored, strong-fi eld laser pulses," Science, vol. 292, pp. 709-713, 2001.

[7] V. Ramakrishna, M. Salapaka, M. Dahleh, and H. Rabitz, 'Controllability of molecular systems," Phys. Rev. A, vol. 51, no. 2, pp. 960-966, 1995.

[8] G. Turinici and H. Rabitz, "Quantum wavefunction controllability," Chem. Phys., vol. 267, pp. 1-9, 2001.

[9] C. Altafi ni, 'Controllability of quantum mechanical systems by root space decomposition of su(n)," Journal of Mathematical Physics, vol. 43, no. 5, pp. 2051-2062, 2002.

[10] F. Albertini and D. D'Alessandro, 'Notions of controllability for bilinear multilevel quantum systems," IEEE Transactions on Automatic Control, vol. 48, no. 8, pp. 1399 - 1403, 2003.

[11] R. Kosloff, S. Rice, P. Gaspard, S. Tersigni, and D. Tannor, "Wavepacket dancing: Achieving chemical selectivity by shaping light pulses." Chemical Physics, vol. 139, pp. 201-220, 1989.

[12] P. Gross, H. Singh, H. Rabitz, K. Mease, and G. Huang, 'Inverse quantum-mechanical control: A means for design and a test of intuition," Phys. Rev. A, vol. 47, p. 4593, 1993.

[13] Y. Chen, P. Gross, V. Ramakrishna, H. Rabitz, and K. Mease, 'Competitive tracking of molecular objectives described by quantum mechanics." J. Chem. Phys., vol. 102, pp. 8001-8010, 1995.

[14] H. Rabitz and W. Zhu, "Quantum control design via adaptive tracking," J. Chem. Phys., vol. 119, no. 7, 2003.

[15] M. Sugawara, "General formulation of locally designed coherent control theory for quantum systems." J. Chem. Phys., vol. 118, no. 15, pp. 6784-6800, 2003.

[16] S. Shi, A. Woody, and H. Rabitz, "Optimal control of selective vibrational excitation in harmonic linear chain molecules," J. Chem. Phys., vol. 88, pp. 6870-6883, 1988.

[17] T. Hornung, M. Motzkus, and R. de Vivie-Riedle, "Adapting OCT and using learning-loops to provide experimentally feasible shaping mask patterns," J. Chem. Phys., vol. 115, pp. 3105-3110, 2001.

[18] Y. Maday and G. Turinici, "New formulations of monotonically convergent quantum control algorithms," J. Chem. Phys, vol. 118, no. 18, 2003.

[19] Y. Ohtsuki, G. Turinici, and H. Rabitz, "Generalized monotonically convergent algorithms for solving quantum optimal control problems," J. Chem. Phys., vol. 120, no. 12, pp. 5509-5517, 2004, also published in the Virtual Journal of Ultrafast Science.

[20] W. Zhu and H. Rabitz, "A rapid monotonically convergent iteration algorithm for quantum optimal control over the expectation value of a positive defi nite operator," J. Chem. Phys., vol. 109, pp. 385-391, 1998.

[21] D. Tannor, V. Kazakov, and V. Orlov, 'Control of photochemical branching: Novel procedures for finding optimal pulses and global upper bounds," in Time Dependent Quantum Molecular Dynamics, Broeckhove J. and Lathouwers L., Eds. Plenum, 1992, pp. 347-360.

[22] S. Schirmer, M. Girardeau, and J. Leahy, "Effi cient algorithm for optimal control of mixed-state quantum systems," Phys. Rev. A, vol. 61, p. 012101,2000

[23] Y. Ohtsuki, W. Zhu, and H. Rabitz, 'Monotonically convergent algorithm for quantum optimal control with dissipation," J. Chem. Phys., vol. 110, pp. 9825-9832, 1999.
[24] Y. Maday, J. Salomon, and G. Turinici, "On the convergence of monotonic algorithms in quantum control," in preparation, 2005.

[25] _ _ 'Parallel in time controls for quantum systems," in preparation, 2005. 